\title{
Lattice QCD on non-orientable manifolds
}

\author{
Simon Mages*, ${ }^{a b}$ Bálint C. Tóth*, ${ }^{c}$ Szabolcs Borsányi, ${ }^{c}$ Zoltán Fodor, ${ }^{a c d}$ Sándor D. \\ Katz, ${ }^{d e}$ and Kálmán K. Szabóc ${ }^{c}$ \\ ${ }^{a}$ Jülich Supercomputing Centre, Jülich D-52425, Germany \\ ${ }^{b}$ University of Regensburg, Department of Physics, Regensburg D-93053, Germany \\ ${ }^{c}$ University of Wuppertal, Department of Physics, Wuppertal D-42097, Germany \\ ${ }^{d}$ Eötvös University, Budapest 1117, Hungary \\ eMTA-ELTE "Lendulet" Lattice Gauge Theory Research Group, Budapest, 1117, Hungary \\ E-mail: simon-wolfgang.magesaur.de, tothbalintaszofi.elte.hu, \\ borsanyi@uni-wuppertal. de, fodorabodri.elte.hu, katzabodri.elte.hu, \\ szabokalgeneral .elte.hu
}

A common problem in lattice QCD simulations on the torus is the extremely long autocorrelation time of the topological charge, when one approaches the continuum limit. The reason is the suppressed tunneling between topological sectors. The problem can be addressed by replacing the torus with a different manifold. Here we propose to use a non-orientable manifold, and show how to define and simulate lattice QCD on it.

34th annual International Symposium on Lattice Field Theory

24-30 July 2016

University of Southampton, UK

${ }^{*}$ Speaker. 


\section{Introduction}

"Topological Freezing" is one of the big obstacles encountered when simulating lattice QCD on a torus at ever smaller lattice spacings. It leads to extremely long autocorrelation times of important observables when the continuum limit is approached. The prime example of an affected observable is the topological charge $Q$. It is given as the integral

$$
Q=\int_{\mathscr{M}} \mathrm{d}^{4} x q(x)
$$

of the topological charge density

$$
q(x)=\frac{1}{4 \pi^{2}} \varepsilon_{\mu v \rho \sigma} \operatorname{tr}\left(F_{\mu v}(x) F_{\rho \sigma}(x)\right)
$$

over the manifold $\mathscr{M}$ of space-time. Usually $\mathscr{M}$ is simply the torus $\mathbb{T}^{4}$. On a finite torus in the continuum this topological charge is discretized for every given gauge configuration. It can only take on values from an equidistant spectrum and is normalized such that it assumes integer values. The charge is a topological invariant meaning that a small change in the field configuration cannot change the value of $Q$. The discretization of the observable means that the field space is separated into a countably infinite number of disconnected sectors labeled by the topological charge. For any finite lattice spacing these properties are realized only up to lattice artefacts and the sectors are separated by a finite action barrier. On the way to the continuum limit, however, this barrier grows large to give the infinite action barrier in the continuum. In particular it grows fast enough to cause trouble for the usual Monte Carlo algorithms which perform small steps in field space with a corresponding small change in the action: the integrated autocorrelation time $\tau_{\text {int }}$ of modes coupled to the topological charge diverges, the different sectors of field space cannot be sampled for affordable Monte Carlo stream lengths and ergodicity is lost. This leads to systematical errors in the measurement of observables which cannot be estimated from the data.

The main idea to be explored in these proceedings is to use instead of the torus $\mathbb{T}^{4}$ a different space-time manifold $\mathscr{M}$ which is non-orientable. The reasoning to experiment with that goes like this: Topological freezing is rooted in the topological structure of field space. The topological sectors which build this structure are labeled by a pseudoscalar quantity, the topological charge $Q$. Furthermore, a pseudoscalar transforms non-trivially under the change of orientability of spacetime.

There are several alternative proposals in the literature to deal with topological freezing, like performing fixed charge simulations [1], manually increasing tunnelling with an additional term in the action and reweighting back to the original theory [2], or using subvolumes for measurement, as the topological charge on a subvolume is not discretized [3]. The authors of [4] propose "metadynamics" simulations: They use a simulation history dependent potential in the action, which punishes sticking to a given topological sector, and reweight back to the original theory. In [5] multiscale equilibration is proposed. Here thermalization is done at a coarser lattice spacing where topology still changes frequently and after that prolong the coarse lattice to a fine lattice spacing. Repeating this gives an ensemble of fine configurations with the well sampled topological charge distribution of the coarse lattice spacing. The most widely accepted method is to use open boundary conditions [6]. 


\section{P-boundaries}

The topological structure of field space depends on the topological structure of the gauge group and on the topological structure of space-time. In the open boundary case the topological structure of space-time is changed by replacing one of the periodic directions with a direction with open boundaries, i.e. with Dirichlet boundary conditions for the fields. This also changes the topology of field space, which can be shown to be connected. The intuitive picture is that the quantized charges localized in instantons and anti-instantons can move freely through the open boundaries of space-time and thereby connect the discrete sectors, whereas on the torus these charges are conserved due to the periodicity of all directions.

While rather elegant in principle, the solution by open boundaries has some additional effects, which - depending on the application - can be problematic. Translational invariance is broken strongly by the boundaries, physics is different at the boundary, and boundary effects propagate into the bulk of the lattice and can contribute systematic deviations to observables. It would therefore be desirable to find a different change in the topology of space-time without any local changes of space-time.

This poses the question of what changes can be made to a manifold without changing the local structure. A quite obvious construction of some possibilities offers itself while constructing a torus from the base manifold by a choice of the boundary conditions. Lets consider the two dimensional case for simplicity. The starting point is a rectangle. Then one can identify the opposite pairs of edges with each other, aka gluing them together to obtain a torus. But during gluing one made a choice not to flip one of the edges before gluing them together. If one flipped one edge once, one would have ended up with a manifold different from the torus, but locally identical to the torus everywhere: the Klein bottle. The most striking feature of the Klein bottle is that it is non-orientable. If one glues together with a flip only one pair of edges and leaves the other pair open, one ends up with a Möbius strip, which is also non-orientable. The torus, the ring which emerges when gluing two sides together without a flip leaving the other sides open, and also the base manifold, the rectangle, are orientable. A manifold is called orientable if one can define consistently a coordinate system with well defined handedness on the complete manifold. This is equivalent to the non-existence of special closed paths on the manifold with the following property: A right-handed system when transported continuously around such a special path becomes a lefthanded system with respect to a stationary system. The concept is illustrated in Fig. 1. Note that locally the closed orientable and non-orientable manifolds are indistinguishable.

Combining this feature with a property of the topological charge and its density leads directly to a candidate for a change of the topology of space-time which could solve the topological freezing problem without tampering with the local structure of space-time: The charge is pseudoscalar, i.e. parity $P$-odd, and therefore transforms under a change of orientation by changing its sign. The sector changing effect of this change can be made obvious in the instanton picture similar to the open boundary case: If one follows the movements of a topological charge density fluctuation around one of the special closed loops on a non-orientable manifold, i.e. along a flipped direction, the sign of the fluctuation changes. If the fluctuation had the charge +1 , it arrives after one roundtrip with a charge of -1 , changing the total charge by -2 . As the movement of the fluctuation around the special path was continuous, the total charge assumed all values between the initial and 

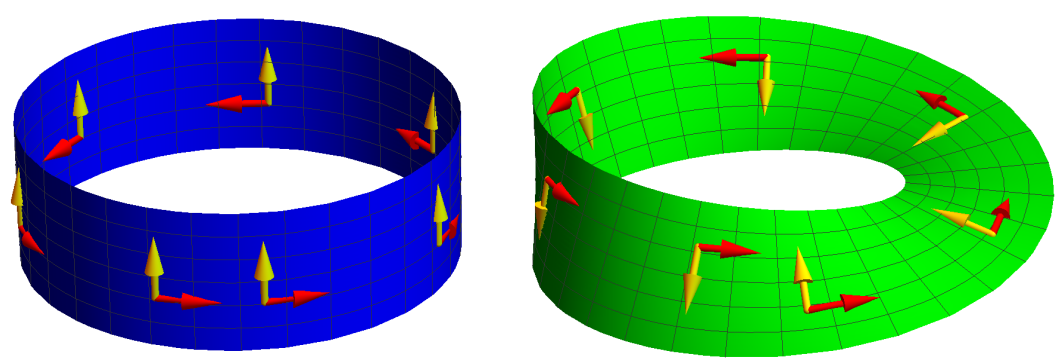

Figure 1: Visualization of a ring as an orientable manifold at the left hand side and a Möbius strip as a non-orientable manifold on the right hand side. On a non-orientable manifold there exist closed paths which change orientation.

final value - assuming the total charge is well defined. The dynamics of the topological charge density is visualized in Fig. 2.
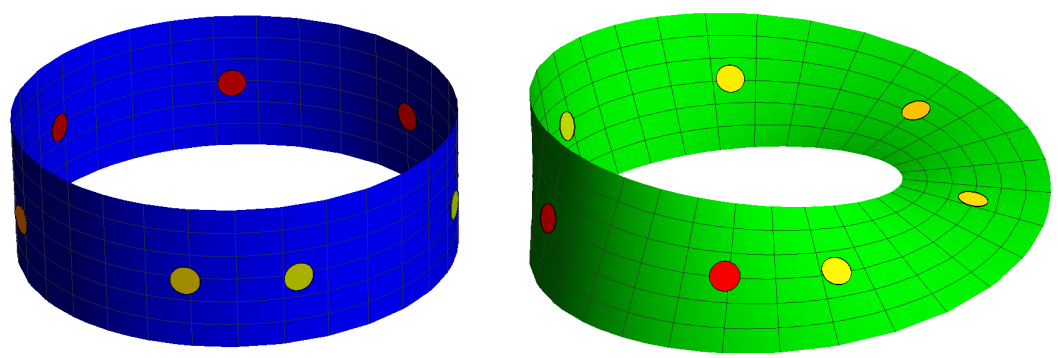

Figure 2: Visualization of the dynamics of a topological charge lump on an orientable manifold on the left hand side and on a non-orientable manifold on the right hand side. For non-orientable manifolds there are paths where the sign of the topological charge lump (visualized by its color) changes its sign.

This is exactly the effect which is exploited in the proposal of $P$-boundaries. They are a specific prescription to change the orientation of the manifold in a physically consistent way. To implement $P$-boundaries one has to

- perform an additional flip while gluing together one of the periodic boundary conditions of the usual torus and

- implement an additional parity transformation $P$ on all fields in the boundary condition.

In this work only the $t$ direction is used as a $P$-periodic direction. Also, instead of a full parity transformation with reflections of all three spacial directions (which are non-trivial to implement on parallel computers), only the single coordinate $x$ is reflected. This is equivalent to a parity transformation followed by a rotation by $180^{\circ}$ which is sufficient for this purpose. In the pure gauge case the transformation of the fields for translations in the $P$-periodic direction is

$$
\begin{aligned}
U_{x}(x, y, z, t+T) & =U_{x}^{\dagger}(L-x-1, y, z, t), \\
U_{i}(x, y, z, t+T) & =U_{i}(L-x, y, z, t)
\end{aligned}
$$


for $i=y, z, t$. In the other three directions the usual periodic boundary condition is kept.

The resulting manifold can be visualized on the universal cover, Fig. 3. The universal cover in the $2 \mathrm{D}$ case is just $\mathbb{R}^{2}$. The base manifold is some rectangle in the universal cover. The boundary conditions yield rules for identification of equivalent points on the universal cover. This figure illustrates the neighbourship relations of points on the base manifold and its replicas in periodic and $P$-periodic directions. In particular it illustrates that there are no locally special points in this construction which can spoil translation invariance and induce large effects on local observables. There are, however, special points along the direction which is reflected: a reflection needs a center of reflection. This point (and distances to this point) can in principle be measured by its effects on particles propagating once around the $P$-periodic direction. If there is a mass gap for $P$-odd propagating states in the theory, then these effects are expected to be exponentially small in the product of the mass gap times the size of the direction.[8]

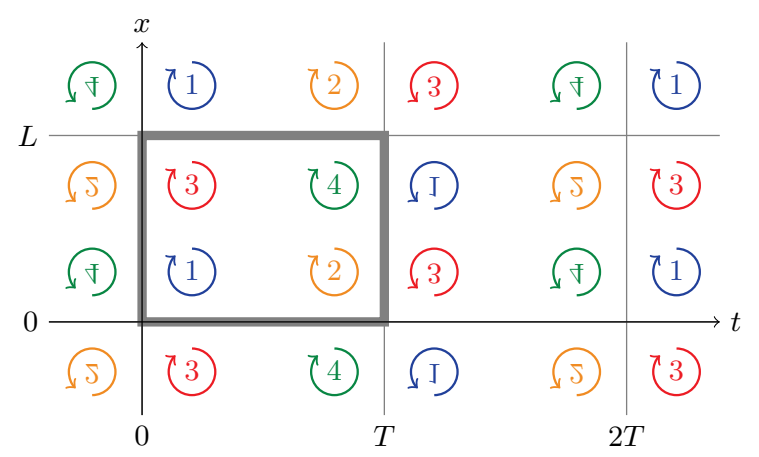

Figure 3: The universal cover for $P$-periodic boundary conditions in time direction for the two dimensional case. The base manifold is the $T \times L$ rectangle. In spatial direction it is replicated periodically, while in $P$-periodic direction it is replicated with a reflection. This reflection is here performed with the line $x=L / 2$ as center. The numbers and colors label identified points of the universal cover including their orientation.

Up to this point the focus of the construction was on the conservation of local symmetries. But there are also important global consequences of the change of orientability. The most important consequence is that integration over the complete manifold cannot be defined via a volume form, as there is no volume form on a non-orientable manifold. One can still construct the integration of scalar densities using a volume element and get the usual expression for the total action from the action density. One cannot, however, define the integration of pseudoscalar densities like the topological charge density $q(x)$ to give a unique meaning to a global topological charge. One can of course define integration of pseudoscalar densities on every orientable submanifold of a non-orientable manifold. This can be used to define a total charge $Q_{m}$ on a maximal oriented submanifold

$$
Q_{m}=\int_{0}^{T} \mathrm{~d} t \int \mathrm{d}^{3} \mathbf{x} q(x),
$$

where a cut in $t$ direction is introduced formally. This cut is only there for the definition of certain observables, which e.g. require the integration of a pseudoscalar over the complete manifold, it is not a physical cut in space-time. In particular, one can define a continuous family of such $Q_{m}$ observables corresponding to all positions of the cut along the $t$ direction. Note that this expression 
for the total charge $Q_{m}$ is the same as for the open boundary case with the important difference, that the cut in that case is physical. For the case with periodic boundary conditions the expression for $Q_{m}$ is exactly the usual total topological charge. As we are mostly interested in comparisons between the periodic, open, and $P$-periodic cases here, we will drop the index $" m "$ and define $Q:=Q_{m}$.

\section{Quenched Data}

After introducing the general idea of QCD on non-orientable manifolds, this section is about numerical tests of $P$-boundaries in the quenched case and their comparison to periodic and open boundaries. For this comparison ensembles of gauge configurations were generated with identical parameters for the three choices of boundary conditions. For the topological freezing the dependence on lattice spacing is the most relevant information. Therefore the physical volume for the runs is kept approximately fixed $L=T \sim 2.27 / T_{c}$, while the lattice spacing is decreased considerably. The gauge action is Symanzik improved and a sweep consists of one heatbath plus four overrelaxation steps. The parameters of the ensembles are given in Tab. 1.

\begin{tabular}{c|c|c|c|c}
$L / a$ & $\beta$ & $w_{0}$ & $a[\mathrm{fm}]$ & $n_{\text {sweep }}$ \\
\hline 16 & 4.42466 & 1.79 & 0.093 & $2 \times 4001$ \\
20 & 4.57857 & 2.24 & 0.075 & $3 \times 4001$ \\
24 & 4.70965 & 2.65 & 0.063 & $4 \times 4001$ \\
32 & 4.92555 & 3.43 & 0.049 & $10 \times 4001$ \\
40 & 5.1 & 4.13 & 0.040 & $19 \times 4001$
\end{tabular}

Table 1: The parameters of the quenched simulations used to compare the different boundary conditions. $L$ is the spatial lattice size, $\beta$ the gauge coupling, $w_{0}$ the Wilson flow based length scale, $a$ the lattice spacing, and $n_{\text {sweep }}$ gives total number of sweeps as product of the number of sweeps between measurements and the number of measurements.

A first qualitative impression can be taken from the history of the topological charge $Q$. Such a comparison is given in Fig. 4. One can readily see from the figure that at the lattice spacing shown the typical symptom for topological freezing on periodic boundaries appears: many consecutive updates without changes in topology. For open and $P$-periodic boundaries the charge changes much more frequently than for periodic boundaries and does not show visible quantization of $Q$. This can be substantiated by a plot of the corresponding histogram of the charges of the same histories, given in Fig. 5: $Q$ is quantized only for periodic boundaries, but not for open or $P$-periodic boundaries.

The next observation concerns the scaling of the integrated autocorrelation time of the topological charge $\tau_{\text {int }}(Q)$ with the lattice spacing. This is given in Fig. 6. It shows that the increase in autocorrelation time with decreasing lattice spacing is largest for the periodic case while the scaling is identical for the open and $P$-periodic setting. For the smallest three lattice spacings in the periodic case the streams were not even long enough to get a good measurement of $\tau_{\text {int }}(Q)$.

The measurements presented above suggest, that open and $P$-periodic boundary conditions show a similar improvement regarding the topological freezing problem. But the motivation to set up QCD on a non-orientable background was not only to reproduce the advantages of open boundaries but also to get rid of local space-time symmetry breaking effects. This is indeed achieved 


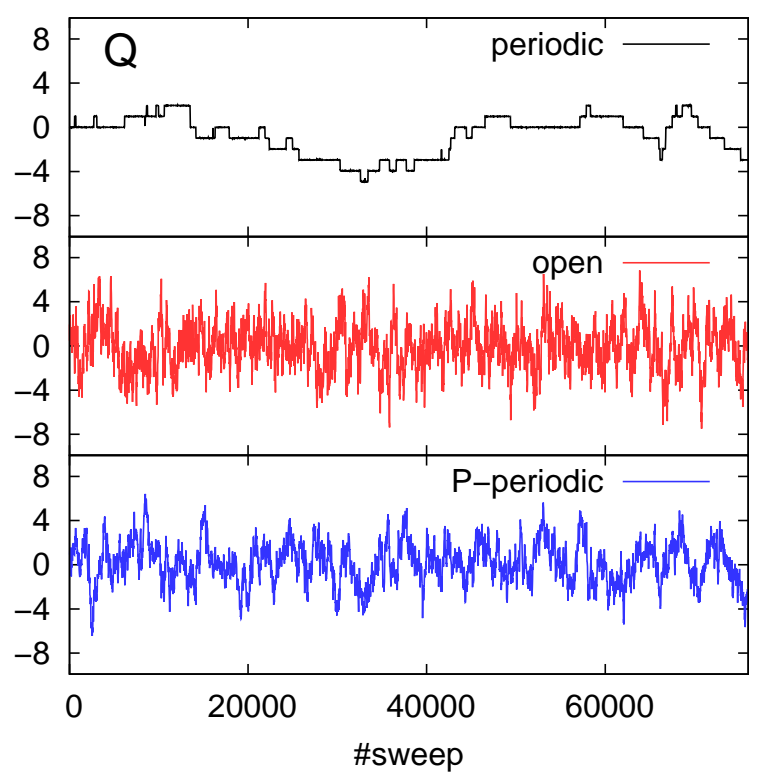

Figure 4: History of the topological charge $Q$ for $\beta=5.1$, lattice spacing $a=0.040 \mathrm{fm}$, and box size $1.6 \mathrm{fm}$.

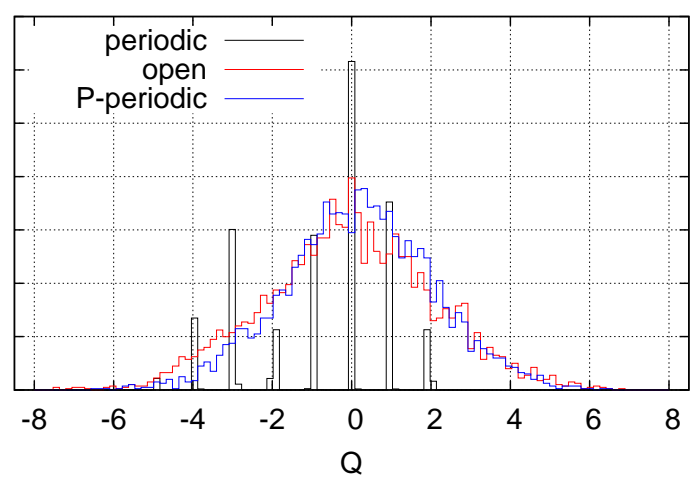

Figure 5: Histogram of the topological charge $Q$ for $\beta=5.1$, lattice spacing $a=0.040 \mathrm{fm}$, and box size $1.6 \mathrm{fm}$.

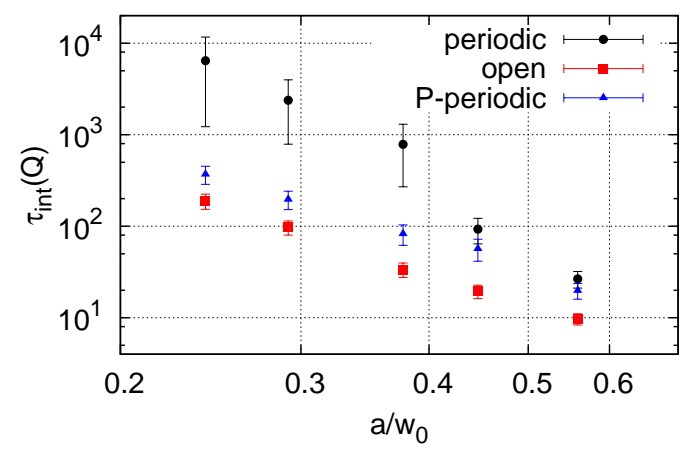

Figure 6: Dependence of the integrated autocorrelation time of the topological charge $\tau_{\text {int }}(Q)$ on the lattice spacing. The box size is $1.6 \mathrm{fm}$. 
and illustrated in Figs. 7 and 8. The figures show measurements in dependence of the time slice at which they are evaluated. The dependence of the action density in Fig. 7 shows that the values for the periodic and $P$-periodic case are within errors consistent with each other and independent of the time slice, while the result for open boundaries shows significant deviations from translational invariance which are especially pronounced near the boundary. For large enough lattices it is expected, that the values from open boundaries become consistent with the ones from periodic boundaries at sufficient distances from the boundary. Fig. 8 displays the measured autocorrelation time of the topological charge per time slice. This also shows significant deviations of translational symmetry for open boundaries, but not for periodic or $P$-periodic boundaries. For the center of the lattice, where boundary effects are small for the open case, open and $P$-periodic lattices show a comparable autocorrelation time, which is much smaller than the autocorrelation time on the periodic lattice.

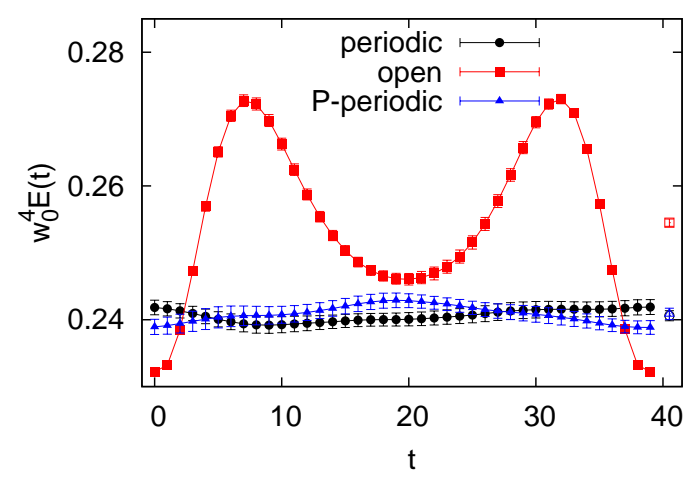

Figure 7: Time slice averaged action density at $\beta=5.1$, lattice spacing $a=0.040 \mathrm{fm}$, and box size $1.6 \mathrm{fm}$. For comparison also the value for the whole lattice is given by the open symbol.

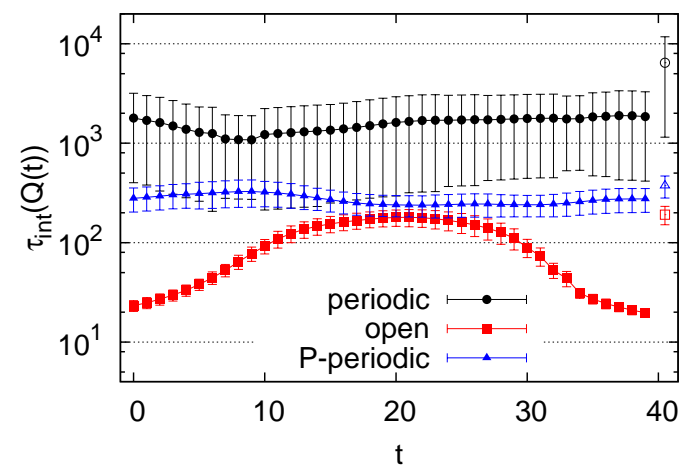

Figure 8: Autocorrelation time of the topological charge of a time slice at $\beta=5.1$, lattice spacing $a=$ $0.040 \mathrm{fm}$, and box size $1.6 \mathrm{fm}$. For comparison also the value for the whole lattice is given by the open symbol.

One might have expected that for this most local observable, the topological charge on a single time slice, the autocorrelation time for the different boundary conditions are the same. That this is different can be seen in the diffusion model of Ref. [7]. In this model the correlations in simulation 
time and euclidean time are described by a diffusion equation

$$
\begin{aligned}
C\left(t, t_{0}, \tau\right) & \equiv\left\langle Q\left(t+t_{0}, \tau_{0}+\tau\right) Q\left(t_{0}, \tau_{0}\right)\right\rangle \\
\frac{\partial}{\partial \tau} C\left(t, t_{0}, \tau\right) & =D \frac{\partial^{2}}{\partial t^{2}} C\left(t, t_{0}, \tau\right)-\frac{1}{\tau_{\text {tunn }}} C\left(t, t_{0}, \tau\right),
\end{aligned}
$$

where $Q(t, \tau)$ is the topological charge on a time slice $t$ at simulation time $\tau, D$ a diffusion constant, and $\tau_{\text {tunn }}$ the timescale for topological charge tunneling. The solutions of this equation determine the integrated autocorrelation time and are determined by the symmetries of the boundaries (and initial conditions). All cases considered here give even solutions in $t$ and the boundary condition determines the (anti-)periodicity of the solution in $t$ as given in Tab. 2. For open boundaries antiperiodicity or periodicity of the solution can be used only at the center of the lattice, as the two nodes of the correlation at the boundary of the lattice can be formally taken as the nodes of an antiperiodic solution. As the center of the lattice is the least affected by boundary effects this suffices for the comparison here. Then both open and $P$-periodic settings give antisymmetric solutions for the correlation function, while the periodic case shows a symmetric solution. In the interesting limit of large tunneling time scale $\tau_{\text {tunn }}$ the zero mode allowed in the symmetric solution of the periodic case leads to the domination of $\tau_{\text {int }}$ by $\tau_{\text {tunn }}$. This shows that even for the autocorrelation of time slices the topological freezing hits $\tau_{\text {int }}$ via $\tau_{\text {tunn }}$ - which is what is seen qualitatively in Fig. 8. The two settings with asymmetric solution have no zero mode in the solution and their autocorrelation time is in the same limit dominated by the diffusion of the topological charge and thus does not diverge along with $\tau_{\text {tunn }}$. The diffusion of topological charge is not hindered even by the existence of exact topological sectors like in the continuum, so that - within this model the topological freezing problem defined as a diverging autocorrelation time of topological charge density is solved for open and $P$-periodic boundaries.

\begin{tabular}{c|c|c} 
Boundary & Periodicity & $\tau_{\text {int }}$ \\
\hline Torus & $C\left(t+T, t_{0}, \tau\right)=C\left(t, t_{0}, \tau\right)$ & $\propto \tau_{\text {tunn }}$ \\
P & $C\left(t+T, t_{0}, \tau\right)=-C\left(t, t_{0}, \tau\right)$ & $\propto D^{-1}$ \\
Open $t_{0}=\frac{T}{2}$ & $" C\left(t+T, \frac{T}{2}, \tau\right)=-C\left(t, \frac{T}{2}, \tau\right) "$ & $\propto D^{-1}$
\end{tabular}

Table 2: Symmetry of the solution of the diffusion model for the different boundary conditions and the resulting limiting behaviour of $\tau_{\text {int }}$ for large $\tau_{\text {tunn }}$.

\section{Fermions}

When we introduce fermions in the simulations with $P$-periodic boundary condition, the reflection in the $x$ coordinate at the timelike boundary has to be applied to the fermion fields as well:

$$
\psi(x, y, z) \longrightarrow i \gamma_{5} \gamma_{x} \psi(L-x, y, z)
$$

Then the factor $\gamma_{5} \gamma_{x}$ appears in the boundary terms, which ruins the $\gamma_{5}$-Hermiticity of the Dirac operator. As a consequence, the fermion determinant will be complex in general, making simulations with algorithms based on importance sampling impossible. 
One possible solution to this situation is to include a charge conjugation additionally to the $x$ reflection: use $C P$-periodic instead of $P$-periodic boundary conditions. Since the topological charge density is not only $P$-odd, but also $C P$-odd, the inclusion of $C$ does not spoil the advantageous features of the $P$-periodic boundary conditions.

The charge conjugation acts on the fermion and gauge fields as

$$
\psi \rightarrow C \bar{\psi}^{T}, \quad \bar{\psi} \rightarrow-\psi^{T} C \quad \text { and } \quad U_{\mu} \rightarrow U_{\mu}^{*},
$$

respectively, where $C=i \gamma_{y} \gamma_{t}$ is the charge conjugation matrix, and the asterisk denotes taking the complex conjugate of each element of $U$.

\subsection{Majorana basis}

Charge conjugation swaps $\psi$ with $\bar{\psi}$, therefore, the fermionic action $S=\bar{\psi} D \psi$ has to be rewritten in a form which treats $\psi$ and $\bar{\psi}$ similarly. To achieve this we express the spinor fields in the Majorana basis [8]: introduce the 8-component spinor fields

$$
\eta=\left(\begin{array}{c}
\eta_{1} \\
\eta_{2}
\end{array}\right)=\frac{1}{\sqrt{2}}\left(\begin{array}{c}
\psi+C \bar{\psi}^{T} \\
-i \psi+i C \psi^{T}
\end{array}\right)=V\left(\begin{array}{c}
\psi \\
\bar{\psi}^{T}
\end{array}\right), \quad \text { where } \quad V=\frac{1}{\sqrt{2}}\left(\begin{array}{cc}
1 & C \\
-i & i C
\end{array}\right) .
$$

Charge conjugation acts particularly simply in this basis:

$$
\eta \rightarrow\left(\begin{array}{c}
\eta_{1} \\
-\eta_{2}
\end{array}\right)=\rho_{3} \eta, \quad \text { where } \rho_{3}=\left(\begin{array}{cc}
1 & 0 \\
0 & -1
\end{array}\right)
$$

that is, $\eta_{1}$ and $\eta_{2}$ are eigenvectors of charge conjugation with eigenvalues 1 and -1 , respectively.

The single flavor action in this basis takes the form

$$
S=\bar{\psi} D \psi=-\frac{1}{2} \eta^{T}\left(V^{-1}\right)^{T}\left(\begin{array}{c}
D^{T} \\
-D
\end{array}\right)\left(V^{-1}\right) \eta=-\frac{1}{2} \eta^{T} C \hat{D} \eta
$$

where

$$
\hat{D}=\frac{1}{2}\left(\begin{array}{cc}
D[U]+C D[U]^{T} C & i D[U]-i C D[U]^{T} C \\
-i D[U]+i C D[U]^{T} C & D[U]+C D[U]^{T} C
\end{array}\right) .
$$

Let us introduce the hatted gauge links

$$
\hat{U}=\left(\begin{array}{cc}
\operatorname{Re}(U) & -\operatorname{Im}(U) \\
\operatorname{Im}(U) & \operatorname{Re}(U)
\end{array}\right)=\left[\operatorname{Re}(U) \cdot 1_{2 \times 2}-i \operatorname{Im}(U) \cdot \rho_{2}\right],
$$

where $\operatorname{Re}(U)$ and $\operatorname{Im}(U)$ denote the matrices obtained by taking the real and imaginary part of each component of $U$, respectively.

The Wilson Dirac operator satisfies $C D[U]^{T} C=D\left[U^{*}\right]$, and is linear in the links, therefore, when Wilson fermions are used, the hatted Dirac operator can be written in the form

$$
\hat{D}=D[\hat{U}] .
$$

That is, $\hat{D}$ can be obtained from the original Dirac operator $D$ by simply replacing the links $U$ with the $6 \times 6$ real matrices $\hat{U}$. Since the mapping $U \mapsto \hat{U}$ is a representation of the group $S U(3)$, equation (4.8) is valid not only for Dirac operators that are linear in the links, but is also valid for operators that are linear in products of links, e.g. the clover improved Wilson Dirac operator. Equation (4.8) is also valid in some more general cases, e.g. in the case of the overlap operator. 


\subsection{CP-periodic boundary}

The most transparent way to construct the $C P$-periodic boundary on the manifold $\mathscr{M}$ is to work on its orientable double cover $2 \mathscr{M}$. Let $2 \mathscr{M}$ be a four dimensional torus of size $L^{3} \times 2 T$, with periodic boundary conditions in all directions for both the gauge fields and the fermionic fields. We consider only those gauge field configurations on $2 \mathscr{M}$, which satisfy the identities

$$
\begin{aligned}
& U_{x}(x, y, z, t+T)=U_{x}^{T}(L-x-1, y, z, t), \\
& U_{i}(x, y, z, t+T)=U_{i}^{*}(L-x, y, z, t), \quad i=y, z, t .
\end{aligned}
$$

The restriction of these gauge configurations onto the base manifold $\mathscr{M}$ are in a one-to-one correspondence with the gauge configurations on $\mathscr{M}$ satisfying the $C P$-periodic boundary conditions.

If $\mathbf{D}$ is a Dirac operator on $2 \mathscr{M}$ that satisfies $\gamma_{5} \mathbf{D} \gamma_{5}=\mathbf{D}^{\dagger}$, then the Dirac operator on the base manifold $\mathscr{M}$ will be given through a projection $\mathbf{P}$, which projects onto the subspace of fermion fields satisfying the desired $C P$-periodic boundary condition: ${ }^{1}$

$$
D_{x, y}=2(\mathbf{P D P})_{x, y} \quad x, y \in \mathscr{M}
$$

In order to describe the projection $\mathbf{P}$, introduce a geometric transformation $\tau$ on $2 \mathscr{M}$, which performs a shift with $T$ in the time direction, and a reflection in $x$ :

$$
\tau\left(\begin{array}{l}
x \\
y \\
z \\
t
\end{array}\right)=\left(\begin{array}{ll}
L-x & (\bmod L) \\
y \\
z \\
t+T & (\bmod 2 T)
\end{array}\right)
$$

Then the transformation we take for the fermionic fields is

$$
(\mathbf{T} \eta)(x)=-\gamma_{5} \gamma_{x} \rho_{2} \rho_{3} \eta(\tau x),
$$

which performs a shift in the time direction, a reflection in the $x$ direction, and a charge conjugation, as illustrated in Fig. 9.

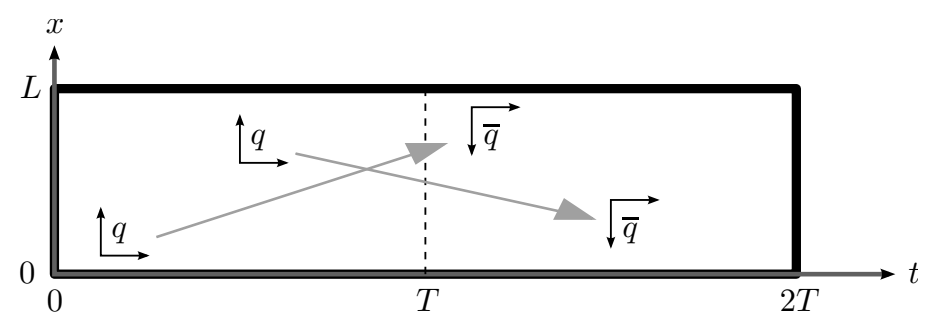

Figure 9: Transformation $\mathbf{T}$ on the doubled manifold $2 \mathscr{M}$ : the fermionic fields are shifted by $T$ in the time direction, reflected in coordinate $x$, and are charge conjugated.

Since $\mathbf{T}$ satisfies

$$
\mathbf{T}^{\dagger}=\mathbf{T}, \quad \mathbf{T}^{2}=\mathbf{1}, \quad[\hat{\mathbf{D}}, \mathbf{T}]=0,
$$

\footnotetext{
${ }^{1}$ Let us fix the notation such that bold faced letters denote operators on the doubled manifold $2 \mathscr{M}$, while letters in italics denote operators on the base manifold $\mathscr{M}$.
} 
we can define the projections $\mathbf{P}_{ \pm}=\frac{\mathbf{1} \pm \mathbf{T}}{2}$, which select either the +1 or the -1 eigenspace of $\mathbf{T}$. Then we define the Dirac operator $\hat{D}_{ \pm}$on $\mathscr{M}$ to be equal to $\mathbf{P}_{ \pm} \hat{\mathbf{D}} \mathbf{P}_{ \pm}$restricted to the range of $\mathbf{P}_{ \pm}$. That is, our Dirac operator on $\mathscr{M}$ is defined as

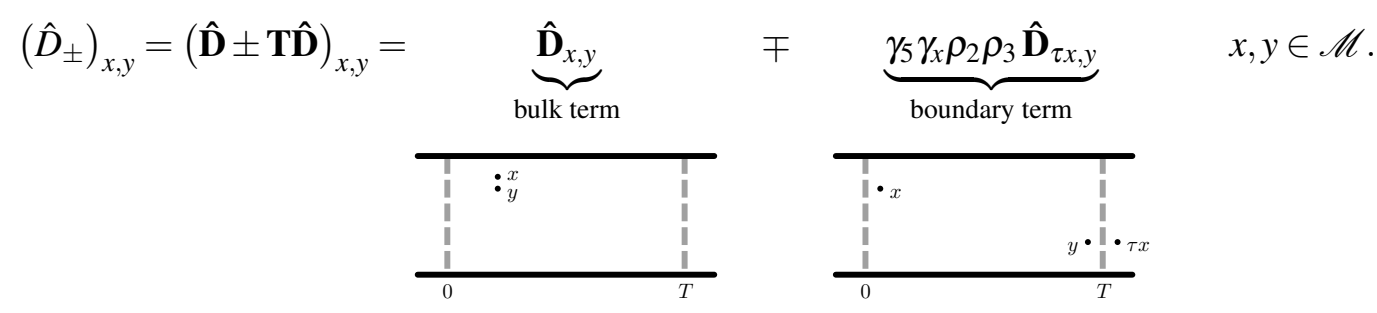

The bulk term connects fermionic fields at neighboring sites away from the $C P$-periodic boundary, while the boundary term connects fermionic fields across the $C P$-periodic boundary. As will be seen from the next subsection, the two choices $\hat{D}_{+}$and $\hat{D}_{-}$are equivalent.

\subsection{Positivity of the action}

The integral of the fermionic action in the Majorana basis

$$
\int \mathrm{d} \eta \exp \left(-\frac{1}{2} \eta^{T} C \hat{D}_{ \pm} \eta\right)=\operatorname{pf}\left(C \hat{D}_{ \pm}\right)
$$

is expressed through the Pfaffian of $C \hat{D}_{ \pm}$. Since it is an antisymmetric matrix, we have

$$
\operatorname{pf}\left(C \hat{D}_{ \pm}\right)^{2}=\operatorname{det}\left(C \hat{D}_{ \pm}\right)=\operatorname{det}\left(\hat{D}_{ \pm}\right) .
$$

In order to see the positivity of $\operatorname{pf}\left(C \hat{D}_{ \pm}\right)$we need to look at the spectrum of $\hat{D}_{ \pm}$.

Since the Dirac operator $\hat{\mathbf{D}}$ on $2 \mathscr{M}$ commutes with $\rho_{2}$ and is $\gamma_{5}$-Hermitian, it is also $\gamma_{5} \rho_{2}$ Hermitian [9]

$$
\gamma_{5} \rho_{2} \hat{\mathbf{D}} \gamma_{5} \rho_{2}=\hat{\mathbf{D}}^{\dagger} \text {. }
$$

In addition, the transformation $\mathbf{T}$ defining the boundary condition commutes with $\gamma_{5} \rho_{2}$, therefore, the projections $\mathbf{P}_{ \pm}$preserve $\gamma_{5} \rho_{2}$-Hermiticity. Thus, the determinant of $\hat{D}_{ \pm}$, which is equal to $\mathbf{P}_{ \pm} \hat{\mathbf{D}} \mathbf{P}_{ \pm}$restricted to the range of $\mathbf{P}_{ \pm}$, is real.

The transformation $\mathbf{T}$ anticommutes with $\rho_{2}$, which has the consequence that $\rho_{2}$ acts as a similarity transformation between $\mathbf{P}_{+} \hat{\mathbf{D}} \mathbf{P}_{+}$and $\mathbf{P}_{-} \hat{\mathbf{D}} \mathbf{P}_{-}$. Therefore, the spectrum of $\hat{\mathbf{D}}$ is equal to the spectrum of $\hat{D}_{ \pm}$with a multiplicity of two. Since $\hat{\mathbf{D}}$ is a usual Wilson-type Dirac operator on a torus with usual periodic boundary conditions, the real parts of all of its eigenvalues are positive close to the continuum. Therefore, the determinant of $\hat{D}_{ \pm}$is positive, the real parts of all of its eigenvalues are positive, and the eigenvalues are either real or come in complex conjugate pairs. As a consequence, $\operatorname{pf}\left(C \hat{D}_{ \pm}\right)>0[8]$.

\subsection{Computing correlators}

In order to compute correlation functions of fermionic variables, we need to express the operators in the Majorana basis. As an example, the interpolating operators for the charged pion are

$$
\begin{gathered}
\mathscr{O}_{\pi^{-}}=\bar{\psi}_{u} \gamma_{5} \psi_{d}=-\frac{1}{2} \eta_{u}^{T} \gamma_{5} C\left(1-\rho_{2}\right) \eta_{d}, \\
\overline{\mathscr{O}}_{\pi^{-}}=-\bar{\psi}_{d} \gamma_{5} \psi_{u}=\frac{1}{2} \eta_{d}^{T} \gamma_{5} C\left(1-\rho_{2}\right) \eta_{u} .
\end{gathered}
$$


Then the pion-pion correlation function between $x$ and $y$ becomes

$$
\left\langle\mathscr{O}_{\pi^{-}}(x) \overline{\mathscr{O}}_{\pi^{-}}(y)\right\rangle=-\frac{1}{4}\left\langle\left(\eta_{u}^{T}\right)_{x} \gamma_{5} C\left(1-\rho_{2}\right)\left(\eta_{d}\right)_{x}\left(\eta_{d}^{T}\right)_{y} \gamma_{5} C\left(1-\rho_{2}\right)\left(\eta_{u}\right)_{y}\right\rangle .
$$

In order to obtain the observable that has to be measured on the gauge configurations we need to perform the Grassmann integral for the $\eta$ fields

$$
\frac{\int \mathrm{d} \eta \eta_{i_{1}} \eta_{i_{2}} \eta_{i_{3}} \eta_{i_{4}} \exp \left(-\frac{1}{2} \eta^{T} M \eta\right)}{\int \mathrm{d} \eta \exp \left(-\frac{1}{2} \eta^{T} M \eta\right)}=\frac{1}{8} \sum_{\sigma \in S_{4}} \operatorname{sgn}(\sigma)\left(M^{-1}\right)_{\sigma\left(i_{1}\right), \sigma\left(i_{2}\right)}\left(M^{-1}\right)_{\sigma\left(i_{3}\right), \sigma\left(i_{4}\right)}
$$

with $M=C \hat{D}$. In the isospin limit we obtain

$$
\left\langle\mathscr{O}_{\pi^{-}}(x) \overline{\mathscr{O}}_{\pi^{-}}(y)\right\rangle=\frac{1}{4} \operatorname{Tr}\left[\left(\hat{D}^{-1}\right)_{y, x} \rho_{2}\left(\hat{D}^{-1}\right)_{x, y}^{\dagger} \rho_{2}+\left(\hat{D}^{-1}\right)_{y, x}\left(\hat{D}^{-1}\right)_{x, y}^{\dagger}\right] .
$$

As an exploratory study, we have computed the pion propagator on $16^{3} \times 32$ quenched configurations generated with $C P$-periodic boundary condition at $\beta=4.35\left(w_{0}=1.57\right)$. We applied 4 steps of stout smearing with $\rho=0.125$, and used the Wilson Dirac operator with bare quark mass $m_{0}=-0.16$. The propagator is shown in the upper panel of Fig. 10. The figure shows that the backward propagation of the pion is suppressed. This suppression can be better understood if we express the pion propagator using the quark propagator $\hat{\mathbf{D}}^{-1}$ acting on $2 \mathscr{M}$ :

$$
\left\langle\mathscr{O}_{\pi^{-}}(x) \overline{\mathscr{O}}_{\pi^{-}}(y)\right\rangle=\frac{1}{2} \operatorname{Tr}\left[\left(\hat{\mathbf{D}}^{-1}\right)_{y, x}\left(\hat{\mathbf{D}}^{-1}\right)_{x, y}^{\dagger}\right] .
$$

The terms containing $\mathbf{T}$ cancel, the sources mirrored into the second half of $2 \mathscr{M}$ do not contribute.

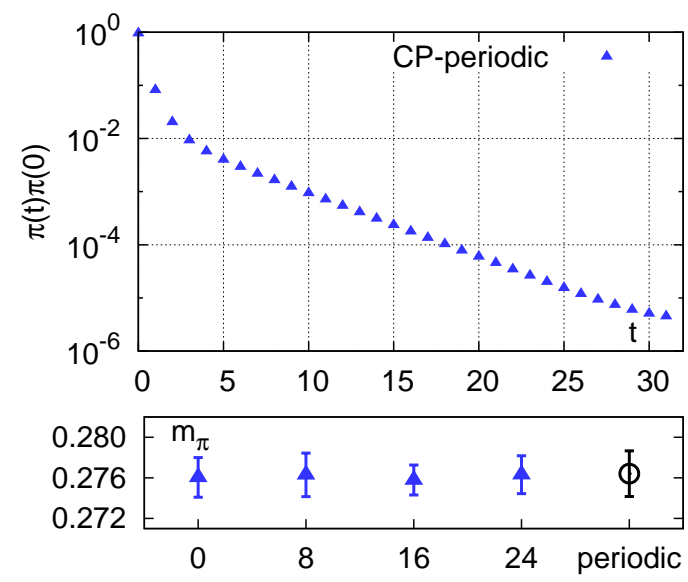

Figure 10: Upper: Pion propagator with $C P$-periodic boundary condition. Lower: Testing translation invariance: Pion masses obtained after shifting the gauge field by $0,8,16$, and 24 units in t-direction (filled), and with periodic boundary condition (open).

\section{Discussion and Outlook}

In these proceedings we have presented the idea to formulate lattice QCD on a non-orientable background via $P$ - and $C P$-periodic boundary conditions. ${ }^{2}$ We have shown in the quenched setting

\footnotetext{
${ }^{2}$ Some results present in these proceedings are published in Ref. [10].
} 
that this leads to similar effects on the autocorrelation time of observables as using open boundary conditions. The inclusion of fermions is not straightforward and leads to a complex action for $P$-periodic boundaries. The inclusion of a $C$ transformation in the boundary solved this issue. We have shown how to construct fermionic observables on a non-orientable background and as a proofof-principle study measured the pion correlation function on quenched $C P$-periodic configurations. We left the implementation and evaluation of dynamic fermions on non-orientable backgrounds for future work.

While the effect of the non-orientability of the background manifold on the measured autocorrelations is arguably as favourable as the one of open boundary configurations, there is a fundamental difference between them: While the field space is connected in the open case, the non-orientable case still comprises two sectors. This can be seen in the instanton picture where the total topological charge $Q$ is localized in $N_{+}$instantons and $N_{-}$anti-instantons of charge \pm 1 each. Changes of the charge by 2 units are readily possible by transporting one anti-instanton once around a non-orientable direction and effectively making it into an instanton. This means that $Q=N_{+}-N_{-}$is not conserved and not even well defined, but $N_{+}+N_{-}$is conserved mod 2 and there are two sectors left, one with an even and one with an odd number of the sum of instantons and anti-instantons. Lattice artifacts can of course much better lead to an even sampling of these two remaining sectors at much smaller lattice spacings than of the infinite number of sectors on the torus. But there is the possibility that some physically interesting observable couples to this two-sector charge, which would then necessitate the measurement of the sector and a strategy to sample both with their proper weights.

\section{References}

[1] S. Aoki, H. Fukaya, S. Hashimoto and T. Onogi, Phys. Rev. D 76 (2007) 054508 doi:10.1103/PhysRevD.76.054508 [arXiv:0707.0396 [hep-lat]].

[2] R. Kitano and N. Yamada, JHEP 1510 (2015) 136 doi:10.1007/JHEP10(2015)136 [arXiv:1506.00370 [hep-ph]].

[3] R. C. Brower et al. [LSD Collaboration], Phys. Rev. D 90 (2014) no.1, 014503 doi:10.1103/PhysRevD.90.014503 [arXiv:1403.2761 [hep-lat]].

[4] A. Laio, G. Martinelli and F. Sanfilippo, JHEP 1607 (2016) 089 doi:10.1007/JHEP07(2016)089 [arXiv:1508.07270 [hep-lat]].

[5] M. G. Endres, R. C. Brower, W. Detmold, K. Orginos and A. V. Pochinsky, Phys. Rev. D 92 (2015) no.11, 114516 doi:10.1103/PhysRevD.92.114516 [arXiv:1510.04675 [hep-lat]].

[6] M. Luscher and S. Schaefer, JHEP 1107 (2011) 036 doi:10.1007/JHEP07(2011)036 [arXiv:1105.4749 [hep-lat]].

[7] G. McGlynn and R. D. Mawhinney, Phys. Rev. D 90 (2014) no.7, 074502 doi:10.1103/PhysRevD.90.074502 [arXiv:1406.4551 [hep-lat]].

[8] B. Lucini, A. Patella, A. Ramos and N. Tantalo, JHEP 1602 (2016) 076 doi:10.1007/JHEP02(2016)076 [arXiv:1509.01636 [hep-th]].

[9] M. A. Metlitski, arXiv:1510.05663 [hep-th].

[10] S. Mages, B. C. Toth, S. Borsanyi, Z. Fodor, S. Katz and K. K. Szabo, arXiv:1512.06804 [hep-lat]. 